\title{
The relationship between weight and pulmonary outcomes in overweight and obese people with cystic fibrosis
}

\author{
John Welter ${ }^{1}$, Alison Lennox ${ }^{1}$, Sankaran Krishnan ${ }^{1}$, Christy Kim ${ }^{1}$, Sheila Krishnan ${ }^{2}$, Haley \\ Thompson $^{1}$, Emily Mcallister ${ }^{1}$, Kristen Huang ${ }^{1}$, Kasiemobi Nwaedozie ${ }^{1}$, and Allen Dozor ${ }^{1}$ \\ ${ }^{1}$ New York Medical College \\ ${ }^{2}$ Touro College of Osteopathic Medicine
}

August 4, 2021

\begin{abstract}
Introduction: A major focus in $\mathrm{CF}$ care aims to increase weight gain. Rates of overweight and obese people with $\mathrm{CF}$ have gradually increased over the past decade. Obesity could be a risk for restriction of lung volumes and airway obstruction as well as increase rates of pulmonary exacerbations in people with CF. Methods: Patients 6 years of age and older were categorized into weight categories based on the CDC definitions. A retrospective chart review was conducted to obtain lung function testing and other outcomes. Results: 107 patients with a median age of 20.6 years were included in this analysis. $8.4 \%, 64 \%$, $18 \%$ and $10 \%$ of patients were underweight, normal/healthy weight, overweight and obese respectively. FEV1 and FVC (\% predicted) did not differ between patients with weights in the normal range vs. patients in the overweight/obese categories. Linear regression analysis showed a direct correlation between BMI and FEV1 that continued as BMI entered overweight and obese categories in both pediatric and adult patients. Overweight/obese patients did not have increased rates of pulmonary exacerbations compared to those in the normal/healthy weight category. Conclusion: As CF therapies continue to improve, an increasing number of people with CF are exceeding the CDC's normal weight range. Gaining weight past the normal range does not appear to negatively impact pulmonary health of people with CF. If this trend of increased weight gain continues, it remains to be seen if it will eventually negatively affect lung health.
\end{abstract}

\section{The relationship between weight and pulmonary outcomes in} overweight and obese people with cystic fibrosis

Welter, John J, MD ${ }^{1}$; Lennox, Alison T, MD ${ }^{1}$; Krishnan, Sankaran, MD ${ }^{1}$; Kim, Christy, MD ${ }^{1}$; Krishnan, Sheila, $\mathrm{MPH}^{2}$; Thompson, Haley, $\mathrm{MD}^{1}$; McAllister, Emily, $\mathrm{MS}^{1}$; Huang, Kristen, $\mathrm{BS}^{1}$; Nwaedozie, Kasiemobi, $\mathrm{MD}^{1}$; Dozor, Allen J, MD ${ }^{1}$

Division of Pediatric Pulmonology, New York Medical College, Valhalla, New York

Touro College of Osteopathic Medicine, New York, New York

Funding: None.

Disclosures: None.

Acknowledgements: Mia-Ashley Spad, New York Medical College, Valhalla, NY

Correspondence Author: John J. Welter, MD Division of Pediatric Pulmonology, Allergy, Immunology, and Sleep Medicine, Boston Children's Health Physicians, Maria Fareri Children's Hospital at Westchester Medical Center, and New York Medical College.

Email: john_welter@bchphysicians.org 
Mailing address: Division of Pediatric Pulmonology, NYMC, 40 Sunshine Cottage Road, Valhalla, NY 10595.

Abbreviated title: Weight and pulmonary outcomes in cystic fibrosis

Key words: Body mass index, forced expiratory volume in 1 second, forced vital capacity, pulmonary exacerbations

\section{Abstract}

Introduction: A major focus in $\mathrm{CF}$ care aims to increase weight gain. Rates of overweight and obese people with CF have gradually increased over the past decade. Obesity could be a risk for restriction of lung volumes and airway obstruction as well as increase rates of pulmonary exacerbations in people with CF.

Methods: Patients 6 years of age and older were categorized into weight categories based on the CDC definitions. A retrospective chart review was conducted to obtain lung function testing and other outcomes.

Results: 107 patients with a median age of 20.6 years were included in this analysis. $8.4 \%, 64 \%, 18 \%$ and $10 \%$ of patients were underweight, normal/healthy weight, overweight and obese respectively. FEV1 and FVC (\% predicted) did not differ between patients with weights in the normal range vs. patients in the overweight/obese categories. Linear regression analysis showed a direct correlation between BMI and FEV1 that continued as BMI entered overweight and obese categories in both pediatric and adult patients. Overweight/obese patients did not have increased rates of pulmonary exacerbations compared to those in the normal/healthy weight category.

Conclusion: As $\mathrm{CF}$ therapies continue to improve, an increasing number of people with $\mathrm{CF}$ are exceeding the CDC's normal weight range. Gaining weight past the normal range does not appear to negatively impact pulmonary health of people with CF. If this trend of increased weight gain continues, it remains to be seen if it will eventually negatively affect lung health.

\section{Introduction:}

Cystic fibrosis $(\mathrm{CF})$ is a disease characterized by chronic lung inflammation and malabsorption which may lead to failure to thrive in infancy without treatment ${ }^{1}$. CF was first described in 1938 as Cystic Fibrosis of the Pancreas ${ }^{2}$, and prior to the advent of pancreatic enzyme replacement therapy, infants with CF would often die from malnutrition ${ }^{3}$. Once pancreatic enzyme replacement became available, patients continued to have difficulties gaining weight due to increased metabolic demand from chronic inflammatory lung disease as well as inefficiencies of pancreatic enzyme replacement ${ }^{4}$. Over the years, it has been demonstrated that nutritional status in people with $\mathrm{CF}$, defined as percent predicted weight-for-length or body mass index (BMI), directly correlates with pulmonary function ${ }^{5}$. This has made increased weight gain a major focus within the CF community. The CF Foundation has set a goal for pediatric patients of a BMI in the $50^{\text {th }}$ percentile or greater, with patients defined as being at risk for nutritional failure with a BMI between $10^{\text {th }}-25^{\text {th }}$ percentile, and in nutritional failure with a BMI below the $10^{\text {th }}$ percentile ${ }^{6}$. Recognition of the relationship between BMI and pulmonary function has led to increased efforts to promote weight gain ${ }^{7}$. In 2009, for the first time in the U.S., more than half of all people with CF between 2-19 years of age had a BMI greater than the $50^{\text {th }}$ percentile ${ }^{7}$. By 2017 , this increased to almost $60 \%^{7}$. The median BMI percentile has continued to rise in this population, and with it the proportion in overweight and obese categories has also continued to rise ${ }^{8}$.

Both otherwise healthy children and children with asthma who are obese have a higher forced vital capacity (FVC) compared to normal weight peers and sometimes there is evidence for airflow obstruction, such as reduced $\mathrm{FEV}_{1} / \mathrm{FVC}$ and increased respiratory system resistance estimated with impulse oscillometry ${ }^{9-11}$. In patients with asthma, obesity is associated with disease severity, and in adults, weight reduction has been shown to improve asthma severity and control, pulmonary function, and quality of life $\mathrm{e}^{10,12}$.

The effects of obesity on pulmonary outcomes in people with CF has not been adequately studied. In a study of overweight and obese adults with CF from 2015 to 2017, $\mathrm{FEV}_{1}$ percent predicted was directly related to rising BMI as patients entered the overweight, but not obese, categories ${ }^{13}$. The objectives of this study were 
to assess the relationship between weight categories and pulmonary outcomes including pulmonary function and rates of pulmonary exacerbations in children and adults with $\mathrm{CF}$.

\section{Methods:}

After approval from the New York Medical College Institutional Review Board, a single-center retrospective chart review was performed of patients with CF seen at the Armond V. Mascia, MD CF Center at New York Medical College from July 1, 2014 to June 30, 2019. Patients were included if they were 6 years of age and older, had a sweat chloride greater than 60 or 2 known CF-causing mutations, and were able to meet ATS criteria for adequate spirometry ${ }^{14}$. BMI was calculated as weight $(\mathrm{kg}) /$ height $\left(\mathrm{m}^{2}\right)$. Adults (people over 20 years of age) and children (people 2 to 20 years of age) were classified based on CDC criteria for weight distribution based on BMI percentiles or absolute BMI:

- Underweight: Children with BMI below $5^{\text {th }}$ percentile, adults with BMI below 18.5.

- Normal or Healthy Weight: Children with BMI $5^{\text {th }}$ to less than the $85^{\text {th }}$ percentile, adults with BMI 18.5-24.9.

- Overweight: Children with BMI $85^{\text {th }}$ to less than the $95^{\text {th }}$ percentile, adults with BMI 25.0-29.9.

- Obese: Children with BMI equal to or greater than $95^{\text {th }}$ percentile, adults with BMI 30.0 and above.

The presence of Cystic Fibrosis Related Diabetes (CFRD) was determined based on CF Foundation guidelines ${ }^{15}$. Results from spirometry were expressed as $\%$ predicted values using Global lung initiative norms ${ }^{16}$. Individual $\mathrm{FEV}_{1} \%$ predicted, $\mathrm{FVC} \%$ predicted, and the absolute ratio of $\mathrm{FEV}_{1} / \mathrm{FVC}$ were calculated as the median of the maximum value of each measure per quarter over the entire study. Pulmonary exacerbations were defined as prescription of oral or intravenous antibiotics by the CF clinician for acute respiratory symptoms. Individual annual pulmonary exacerbation rates were calculated by dividing the number of pulmonary exacerbations over the course of the study divided by the number of years the patient participated in the study.

\section{Statistical analysis}

For primary analysis, people in each of the CDC weight categories were compared. Patient characteristics including sex, sweat chloride levels, presence of CFRD, treatment with nutritional therapies, treatment with CFTR modulators and pulmonary outcomes were evaluated for differences among weight categories. These characteristics and CDC weight categories were compared between children (6-19 years of age) and adults (greater than or equal to 20 years of age). Categorical values were summarized by frequencies and were compared using $\chi^{2}$ analysis. Continuous variables were summarized by median and IQR and were compared using the Mann-Whitney rank sum test. For secondary analysis, linear regression was performed in children and adults, separately comparing BMI percentiles and absolute BMI with $\mathrm{FEV}_{1} \%$ predicted and $\mathrm{FVC} \%$ predicted.

All statistical analyses were performed using SigmaPlot 13.0 software (Systat Software Inc, San Jose, California).

\section{Results}

\section{Patient Characteristics}

Patient characteristics and comparison of weight categories are in Table 1. 105 people with CF, 6 years of age or older, seen over the 5-year study period, completed 1,931 clinic visits that were included in this analysis. Median age was 20.5 years (IQR: 11.9, 25.7). 9\%, 64\%, $18 \%$ and $10 \%$ of patients were underweight, normal/healthy weight, overweight and obese, respectively. There were no significant changes in the proportion of patients in each weight category over the 5 years evaluated. Obese patients had significantly lower sweat chloride (71 vs. 88,97 and $91 \mathrm{mmol} / \mathrm{L}, \mathrm{p}=0.008)$ and were less likely to be prescribed pancreatic enzymes ( $30 \%$ vs. $89 \%, 87 \%$ and $95 \%, \mathrm{p}<0.001$ ) than people who were underweight, normal weight and overweight, respectively. More obese patients were prescribed ivacaftor than those with normal/healthy weight (50\% vs. $15 \%$ respectively, $\mathrm{p}=0.02$ ). There was no difference in ivacaftor/tezacaftor or ivacaftor/lumacaftor between 
groups. Underweight people were more likely to have CFRD than normal/healthy weight and obese people ( $56 \%$ vs. $10 \%$ and $0 \%$ respectively, $\mathrm{p}=0.004$ and 0.011 ).

Children and adults are compared in Table 2. $19 \%$ of children and $35 \%$ of adults were characterized as either overweight or obese, $\mathrm{p}=0.062$. Children were more likely to be in the normal/healthy weight category than adults, $\mathrm{p}=0.017$. There were no significant differences in gender, pancreatic enzyme replacement, sweat chloride, rates of CFRD or nutritional supplements between children and adults.

\section{Pulmonary Outcomes}

There were no differences in annual rates of pulmonary exacerbations between weight categories (Table 1). $\mathrm{FEV}_{1} \%$ predicted, $\mathrm{FEV}_{1} / \mathrm{FVC}$ and FEF25-75 \% predicted were lower in people who were underweight than those in the other weight categories (Table 1, Figure 1). Scattergrams of $\mathrm{FEV}_{1} \%$ predicted and BMI are in Figure 2. On linear regression, there was a direct correlation between BMI and $\mathrm{FEV}_{1} \%$ predicted in all weight categories. This was true for both children and adults (Figure $2 \mathrm{a}$ and $2 \mathrm{~b}$ ).

The relationship between BMI and FVC are demonstrated in Figure 3. There was a direct correlation between BMI, percentile and FVC, \% predicted, in both children and adults.

\section{Discussion}

It is well established that pulmonary function and nutritional status, as reflected by BMI, are highly correlated in patients with $\mathrm{CF}^{17}$. Increasing rates of obesity in people with $\mathrm{CF}$ raises concern this may no longer be the case given the detrimental effects of obesity in other chronic lung diseases. Obese people with COPD have decreased lung compliance and reduced small airway flows and obese people with asthma have more frequent and severe exacerbations and are less responsive to treatment ${ }^{18,19}$. Asthma and COPD are similar to CF as they are all chronic obstructive lung diseases; but differ widely in their underlying pathophysiology. Similar to Hanna et. al in a 2015 study, we found underweight people with $\mathrm{CF}$ have lower $\mathrm{FEV}_{1} \%$ predicted when compared to other weight groups ${ }^{20}$. In this single-center study, the positive correlation between BMI and pulmonary function continued even in obese patients. Furthermore, the rates of pulmonary exacerbations were not higher in obese patients.

One might speculate that obesity could result in lung restriction, i.e., decreased FVC, but this was not noted in this study. In people with CF, the greater the BMI, the greater the FVC which is consistent with the literature in healthy children and children with asthma, the reasons for this are unclear ${ }^{11}$. Vital capacity was reduced in underweight people with $\mathrm{CF}$ when compared to the other weight categories. There is no evidence of restrictive lung disease in those who are obese.

While we found people with $\mathrm{CF}$ who are obese have higher $\mathrm{FEV}_{1} \%$ predicted than underweight people with $\mathrm{CF}$, there is no significant increase in $\mathrm{FEV}_{1} \%$ predicted when obese people with $\mathrm{CF}$ were compared to those in the normal or overweight categories. There are multiple potential reasons why people with $\mathrm{CF}$ who are obese have higher $\mathrm{FEV}_{1} \%$ predicted than those who are underweight. Pancreatic enzyme use was significantly lower in the obese group. Pancreatic sufficiency and milder lung disease are associated with lower sweat chloride levels, as was seen in the obese group, likely due to relatively increased CFTR function and subsequently less severe disease ${ }^{21}$. Underweight patients in this analysis were more likely to have CFRD. It is well known that CFRD is associated with decline in lung function as well as decreasing nutritional status in people with $\mathrm{CF}^{22}$. While this study shows continued improvements in parameters associated with respiratory health in people with $\mathrm{CF}$ even as they become obese, there is insufficient evidence to support counseling patients to takes steps to increase weight gain beyond the normal/healthy CDC weight category. It is not clear whether worsening rates, and severity, of obesity might eventually begin to have detrimental effects on pulmonary function in people with $\mathrm{CF}$.

There was a trend towards higher rates of ivacaftor use in obese patients compared to the other weight categories. It is possible that the continued improvement of airway flows and volumes in obese patients could be attributed to potentiation of lung CFTR activity by ivacaftor rather than simply the nutritional benefit. We did not find the same trend in those on ivacaftor/lumacaftor or ivacaftor/tezacaftor. Since 
the end of our study ivacaftor/tezacaftor/elexacaftor has been approved for people with CF who have at least one F508del mutation, and it is possible that almost $90 \%$ of people with CF will have highly effective CFTR modulators (HEM) available for them. People with CF started on HEM have significant increases in weight and it is concerning that this may contribute to a rapid increase in obesity rates among people with $\mathrm{CF}^{23,24}$. Given potential increases in life expectancy with HEM use, these people may be at increased risk for obesity related diseases such as cardiovascular and endocrine disorders. In addition, surprisingly we found $4(14 \%)$ of the 29 patients in the overweight and obese categories are still prescribed supplemental feedings. Obesity increases the risk for co-morbidities seen in the general population such as hypertension, obstructive sleep apnea, Type 2 diabetes, and cancer and can also lead to lower lung transplant survival rates in people with $\mathrm{CF}^{25-27}$. Given the rising rates of obesity in people with $\mathrm{CF}$, revision of nutritional guidelines may be required, especially in people prescribed HEM.

Our study has several limitations. This was a retrospective chart review and the historical data collected was limited by chart documentation. CF center nutritional outcomes vary across the United States and between nations. Since this is a single center study, the proportion of patients categorized as either overweight or obese may not reflect the overall population of people with CF. Outcomes such as supplemental feedings were based on patient plans and may not reflect the actual number of patients adhering to these plans. Since this is a cross sectional study, casual relationships cannot be inferred. BMI was used to classify patients' nutritional status but body composition of body fat and lean muscle mass may vary widely in people with the same BMI and may more directly impact pulmonary outcomes than BMI alone.

\section{Conclusion}

In this single-center study, $28 \%$ of people with CF are overweight or obese. There did not appear to be any adverse effects of obesity on pulmonary function or rate of pulmonary exacerbations over a 4-year period. If the current trend of increased weight gain in people with CF continues, it will remain to be seen if obesity may eventually negatively impact lung health.

\section{References}

1. VanDevanter DR, Kahle JS, O'Sullivan AK, Sikirica S, Hodgkins PS. 2016. Cystic fibrosis in young children: A review of disease manifestation, progression, and response to early treatment. J Cyst Fibros. $15(2): 147-157$.

2. Andersen DH. 1938. Cystic fibrosis of the pancreas and its relation to celiac disease: A clinical and pathologic study. American Journal of Diseases of Children. 56(2):344-399.

3. Dodge JA, Turck D. 2006. Cystic fibrosis: Nutritional consequences and management. Best Pract Res Clin Gastroenterol. 20(3):531-546.

4. Culhane S, George C, Pearo B, Spoede E. 2013. Malnutrition in cystic fibrosis: A review. Nutr Clin Pract. 28(6):676-683.

5. Steinkamp G, Wiedemann B. 2002. Relationship between nutritional status and lung function in cystic fibrosis: Cross sectional and longitudinal analyses from the german cf quality assurance (cfqa) project. Thorax. 57(7):596-601.

6. Lai HJ, Shoff SM. 2008. Classification of malnutrition in cystic fibrosis: Implications for evaluating and benchmarking clinical practice performance. Am J Clin Nutr. 88(1):161-166.

7. Litvin M, Yoon JC, Leey Casella J, Blackman SM, Brennan AL. 2019. Energy balance and obesity in individuals with cystic fibrosis. J Cyst Fibros. 18 Suppl 2:S38-S47.

8. Flume P, Fernandez GS, Schechter MS, Fink A. 2019. Prevalence of obesity in people with cystic fibrosis over a 20-year period. Pediatric Pulmonology. 54:S263

9. Ekström S, Hallberg J, Kull I, Protudjer JLP, Thunqvist P, Bottai M, Gustafsson PM, Bergström A, Melén 
E. 2018. Body mass index status and peripheral airway obstruction in school-age children: A populationbased cohort study. Thorax. 73(6):538-545.

10. Tantisira KG, Weiss ST. 2001. Complex interactions in complex traits: Obesity and asthma. Thorax. 56 Suppl 2:ii64-73.

11. Forno E, Han YY, Mullen J, Celedón JC. 2018. Overweight, obesity, and lung function in children and adults-a meta-analysis. J Allergy Clin Immunol Pract. 6(2):570-581.e510.

12. Eneli IU, Skybo T, Camargo CA. 2008. Weight loss and asthma: A systematic review. Thorax. 63(8):671676.

13. Harindhanavudhi T, Wang Q, Dunitz J, Moran A, Moheet A. 2020. Prevalence and factors associated with overweight and obesity in adults with cystic fibrosis: A single-center analysis. J Cyst Fibros. 19(1):139-145.

14. Miller MR, Hankinson J, Brusasco V, Burgos F, Casaburi R, Coates A, Crapo R, Enright P, van der Grinten CP, Gustafsson P et al. 2005. Standardisation of spirometry. Eur Respir J. 26(2):319-338.

15. Moran A, Brunzell C, Cohen RC, Katz M, Marshall BC, Onady G, Robinson KA, Sabadosa KA, Stecenko A, Slovis B et al. 2010. Clinical care guidelines for cystic fibrosis-related diabetes: A position statement of the american diabetes association and a clinical practice guideline of the cystic fibrosis foundation, endorsed by the pediatric endocrine society. Diabetes Care. 33(12):2697-2708.

16. Quanjer PH, Stanojevic S, Cole TJ, Baur X, Hall GL, Culver BH, Enright PL, Hankinson JL, Ip MS, Zheng J et al. 2012. Multi-ethnic reference values for spirometry for the 3-95-yr age range: The global lung function 2012 equations. Eur Respir J. 40(6):1324-1343.

17. Stallings VA, Stark LJ, Robinson KA, Feranchak AP, Quinton H, Subcommittee CPGoGaN, Group AHW. 2008. Evidence-based practice recommendations for nutrition-related management of children and adults with cystic fibrosis and pancreatic insufficiency: Results of a systematic review. J Am Diet Assoc. 108(5):832-839.

18. Franssen FM, O'Donnell DE, Goossens GH, Blaak EE, Schols AM. 2008. Obesity and the lung: 5. Obesity and copd. Thorax. 63(12):1110-1117.

19. Peters U, Dixon AE, Forno E. 2018. Obesity and asthma. J Allergy Clin Immunol. 141(4):1169-1179.

20. Hanna RM, Weiner DJ. 2015. Overweight and obesity in patients with cystic fibrosis: A center-based analysis. Pediatr Pulmonol. 50(1):35-41.

21. McKone EF, Emerson SS, Edwards KL, Aitken ML. 2003. Effect of genotype on phenotype and mortality in cystic fibrosis: A retrospective cohort study. Lancet. 361(9370):1671-1676.

22. Terliesner N, Vogel M, Steighardt A, Gausche R, Henn C, Hentschel J, Kapellen T, Klamt S, Gebhardt J, Kiess W et al. 2017. Cystic-fibrosis related-diabetes (cfrd) is preceded by and associated with growth failure and deteriorating lung function. J Pediatr Endocrinol Metab. 30(8):815-821.

23. Middleton PG, Mall MA, Dřevínek P, Lands LC, McKone EF, Polineni D, Ramsey BW, Taylor-Cousar JL, Tullis E, Vermeulen F et al. 2019. Elexacaftor-tezacaftor-ivacaftor for cystic fibrosis with a single phe508del allele. N Engl J Med. 381(19):1809-1819.

24. Heijerman HGM, McKone EF, Downey DG, Van Braeckel E, Rowe SM, Tullis E, Mall MA, Welter JJ, Ramsey BW, McKee CM et al. 2019. Efficacy and safety of the elexacaftor plus tezacaftor plus ivacaftor combination regimen in people with cystic fibrosis homozygous for the f508del mutation: A double-blind, randomised, phase 3 trial. Lancet. 394(10212):1940-1948.

25. Guh DP, Zhang W, Bansback N, Amarsi Z, Birmingham CL, Anis AH. 2009. The incidence of comorbidities related to obesity and overweight: A systematic review and meta-analysis. BMC Public Health. 9:88. 
26. Young T, Peppard PE, Gottlieb DJ. 2002. Epidemiology of obstructive sleep apnea: A population health perspective. Am J Respir Crit Care Med. 165(9):1217-1239.

27. Chaikriangkrai K, Jhun HY, Graviss EA, Jyothula S. 2015. Overweight-mortality paradox and impact of six-minute walk distance in lung transplantation. Ann Thorac Med. 10(3):169-175.

\section{Hosted file}

Table 1.docx available at https://authorea.com/users/429112/articles/532852-the-relationshipbetween-weight-and-pulmonary-outcomes-in-overweight-and-obese-people-with-cystic-

fibrosis

\section{Hosted file}

Table 2.docx available at https://authorea.com/users/429112/articles/532852-the-relationshipbetween-weight-and-pulmonary-outcomes-in-overweight-and-obese-people-with-cysticfibrosis

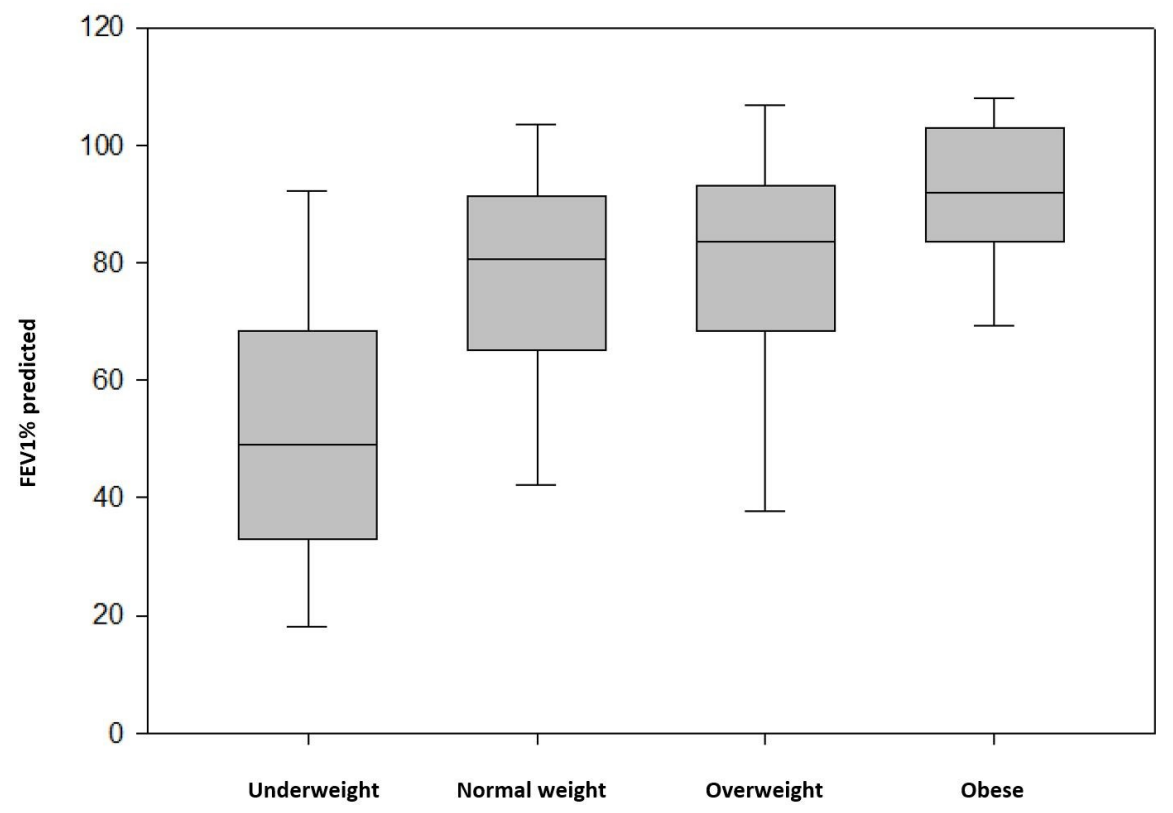


A.
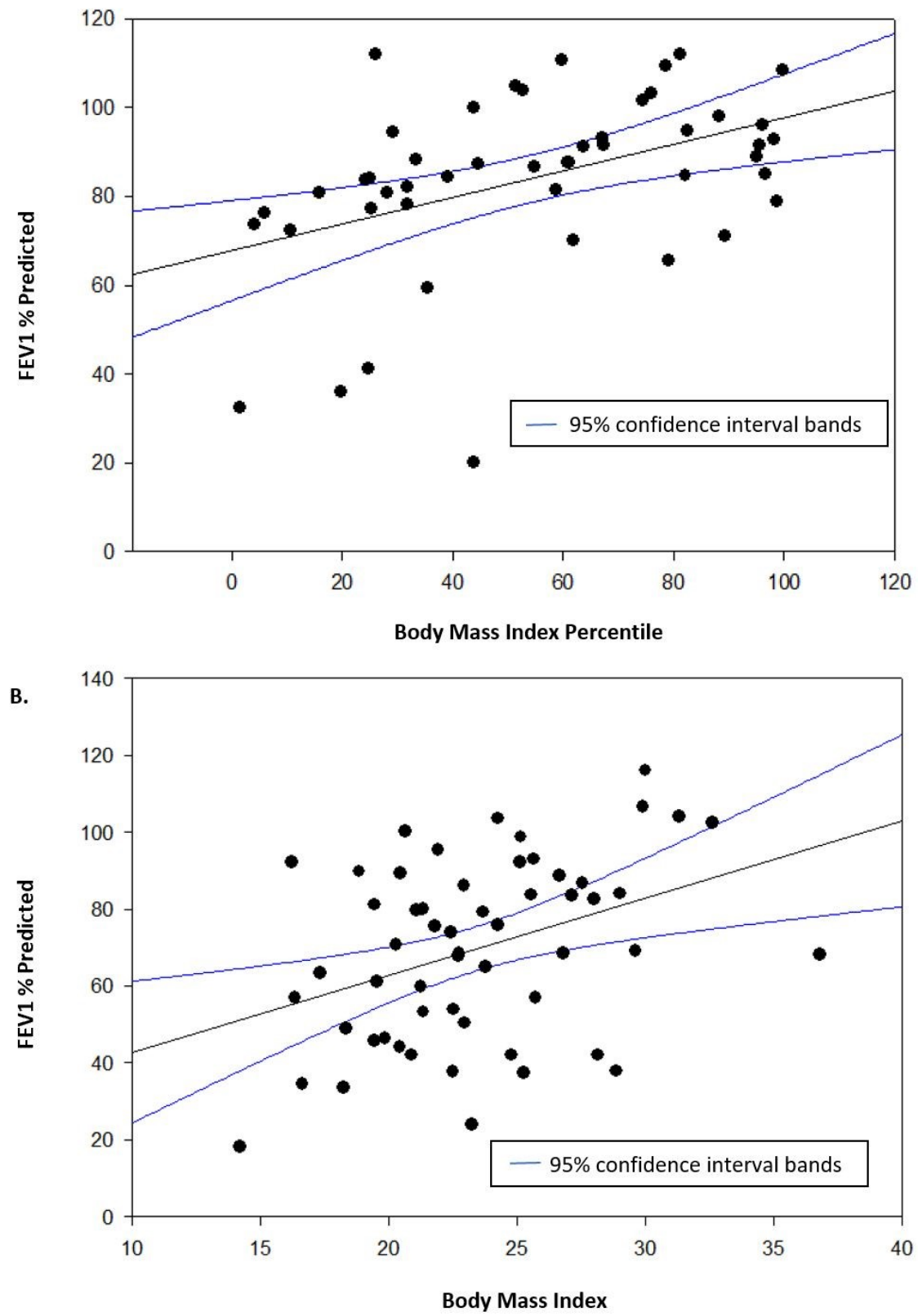
A.
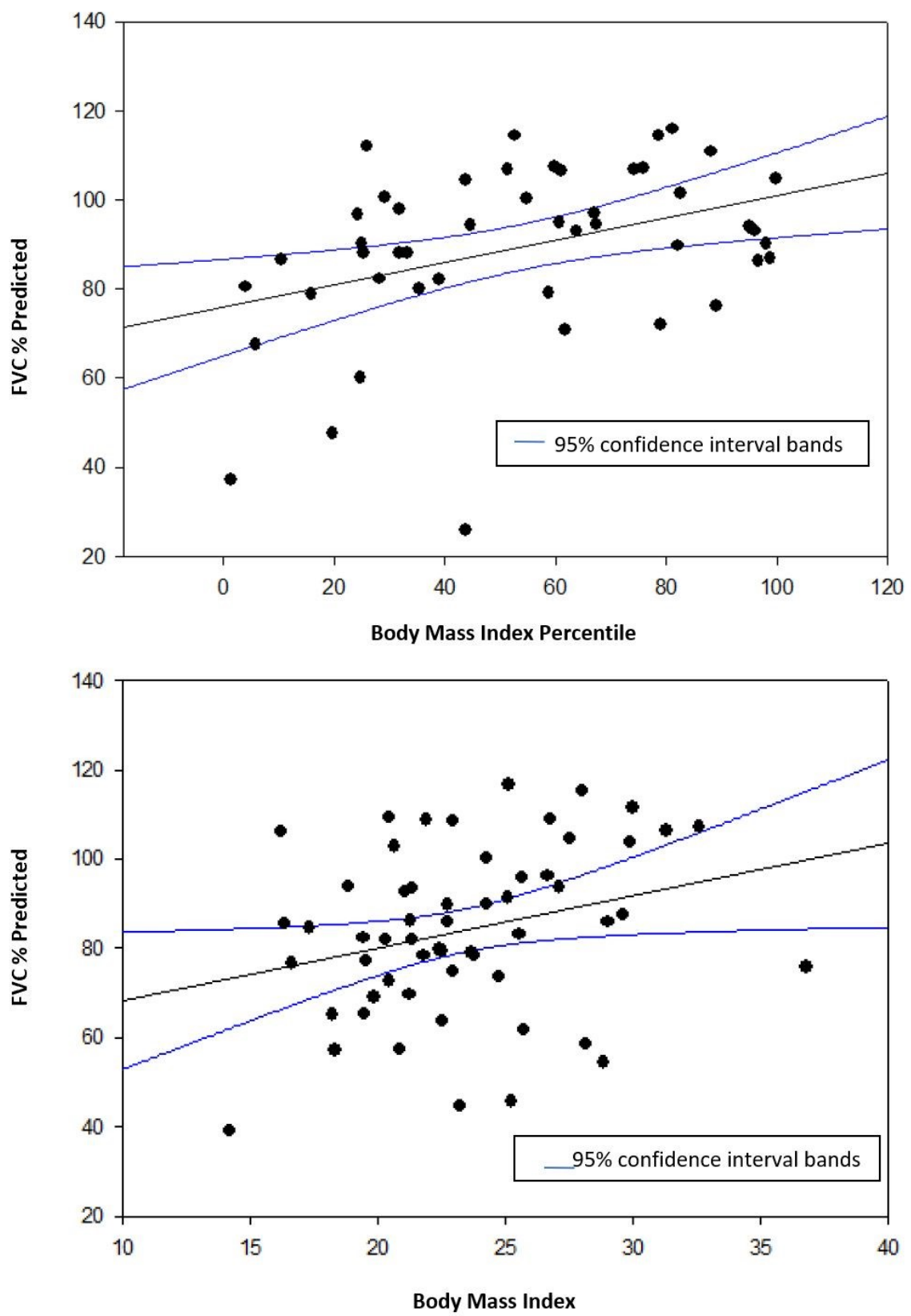